\title{
45.
}

\section{ON THE THEORY OF ELLIPTIC FUNCTIONS.}

[From the Cambridge and Dublin Mathematical Journal, vol. II. (1847), pp. 256-266.]

Adopting the notation of the Fund. Nova, except that for shortness sn $u$, cn $u$, dn $u$ are written instead of $\operatorname{sinam} u, \operatorname{cosam} u, \nabla$ am $u$, let the functions $\Theta(u), \mathrm{H}(u)$ be defined by the equations

$$
\begin{aligned}
\Theta u & =\sqrt{ }\left(\frac{2 K k^{\prime}}{\pi}\right) e^{\frac{1}{2} u^{2}\left(1-\frac{E}{K}\right)-k^{2} \int_{0} d u \int_{0} d u \mathrm{sn}^{2} u} \\
\mathrm{H} u & =-i e^{-\frac{\pi\left(K^{\prime}-2 i u\right)}{4 K}} \Theta\left(u+i K^{\prime}\right)
\end{aligned}
$$

it is required from these equations to express sn $u$ in terms of the functions $\mathrm{H}(u)$, $\Theta(u)$. To accomplish this we have

$$
\begin{aligned}
\frac{d^{2}}{d u^{2}} \log \operatorname{sn} u & =\frac{1}{\operatorname{sn} u} \frac{d^{2}}{d u^{2}} \operatorname{sn} u-\frac{1}{\operatorname{sn}^{2} u}\left(\frac{\bar{d}}{d u} \operatorname{sn} u\right)^{2} \\
& =-\left(1+k^{2}\right)+2 k^{2} \operatorname{sn}^{2} u-\left\{\frac{1}{\operatorname{sn}^{2} u}-\left(1+k^{2}\right)+k^{2} \operatorname{sn}^{2} u\right\} \\
& =k^{2} \operatorname{sn}^{2} u-\frac{1}{\operatorname{sn}^{2} u}
\end{aligned}
$$

whence also

$$
\frac{d^{2}}{d u^{2}} \log \operatorname{sn} u=k^{2} \operatorname{sn}^{2} u-k^{2} \operatorname{sn}^{2}\left(u+i K^{\prime}\right)
$$

If for a moment

$$
\psi, u=\int_{0} d u \operatorname{sn}^{2} u, \quad \psi_{11} u=\int_{0} d u \int_{0} d u \operatorname{sn}^{2} u,
$$

then

$$
\log \operatorname{sn} u=k^{2} \psi_{1 /} u-k^{2} \psi_{\prime \prime}\left(u+i K^{\prime}\right)+A u+B ;
$$


or writing $-u$ for $u$ and subtracting, $\psi_{\|} u$ being an even function,

$$
2 A u=\pi i-k^{2} \psi_{\prime \prime}\left(i K^{\prime}-u\right)+k^{2} \psi_{1 /}\left(i K^{\prime}+u\right),
$$

or putting $u=K$,

$$
2 A K=\pi i-k^{2} \psi_{11}\left(i K^{\prime}-K\right)+k^{2} \psi_{\text {" }}\left(i K^{\prime}+K\right) .
$$

Now

$$
\begin{aligned}
& \operatorname{sn}^{2}(u+K)-\operatorname{sn}^{2}(u-K)=0, \\
& \psi_{1}(u+K)-\psi_{i}(u-K)=2 \quad \psi_{,} K, \\
& \psi_{\text {" }}(u+K)-\psi_{\text {" }}(u-K)=2 u \psi_{\text {, }} \text {; } \\
& \psi_{\text {" }}\left(i K^{\prime}+K\right)-\psi_{\text {" }}\left(i K^{\prime}-K\right)=2 i K^{\prime} \psi_{,}, K .
\end{aligned}
$$

or

Also

$$
E(u)=u-k^{2} \psi, u,
$$

$$
E=K-k^{2} \psi, K, \quad \text { i.e. } \quad \psi, K=\frac{K}{k^{2}}\left(1-\frac{E}{K}\right)
$$

Hence

$$
A=i K^{\prime}\left(1-\frac{E}{K}\right)+\frac{\pi i}{2 K}
$$

$$
\begin{gathered}
\log \operatorname{sn} u=k^{2} \psi_{\text {," }} u-k^{2} \psi_{\text {” }}\left(u+i K^{\prime}\right)+u i K^{\prime}\left(1-\frac{E}{K}\right)+\frac{\pi u i}{2 K}+B \\
=k^{2} \psi_{\text {, }} u-k^{2} \psi_{\text {” }}\left(u+i K^{\prime}\right)+\frac{1}{2}\left[\left(u+i K^{\prime}\right)^{2}-u^{2}\right]\left(1-\frac{E}{K}\right)+\frac{\pi u i}{2 K}+B^{\prime}, \\
\log \operatorname{sn} u=\log \Theta\left(u+i K^{\prime}\right)-\log \Theta u+\frac{\pi u i}{2 K}+B^{\prime},
\end{gathered}
$$

or, changing the constant,

$$
\operatorname{sn} u=C e^{\frac{\pi u i}{2 K}} \frac{\Theta\left(u+i K^{\prime}\right)}{\Theta u} .
$$

Now, to determine $C$, write $u-i K^{\prime}$ for $u$; this gives

$$
\frac{1}{k \operatorname{sn} u}=C e^{\frac{\pi i}{2 K}\left(u-i K^{\prime}\right)} \frac{\Theta u}{\Theta\left(u-i K^{\prime}\right)}=
$$

and again changing $u$ into $-u$,

$$
-\operatorname{sn} u=C e^{-\frac{\pi u i}{2 K}} \frac{\Theta\left(u-i K^{\prime}\right)}{\Theta u}:
$$

whence, multiplying these last two equations,

$$
C^{2}=-\frac{1}{k} e^{-\frac{\pi K^{\prime}}{2 K}},
$$


or

$$
C=\frac{1}{i \sqrt{ } k} e^{-\frac{\pi K^{\prime}}{4 K}}
$$

whence

$$
\text { sn } u=\frac{1}{i \sqrt{ } k} e^{-\frac{\pi\left(K^{\prime}-2 i u\right)}{4 K}} \frac{\Theta\left(u+i K^{\prime}\right)}{\Theta u},
$$

i.e.

$$
\sqrt{ } k \operatorname{sn} u=\frac{\mathrm{H}(u)}{\Theta(u)}
$$

and the equations (1), (2) and (3) may be considered as comprehending the theory of the functions $H(u), \Theta(u)$. The preceding process is, in fact, the converse of that made use of in the Fund. Nova; Jacobi having obtained for sn $u$ an expression in the form of a fraction, takes the numerator of it for $\mathrm{H}(u)$ and the denominator for $\Theta(u)$, and thence deduces the equations (1), (2), the intermediate steps of the demonstration being conducted by means of infinite series; the necessity of which is avoided by the preceding investigation.

I proceed to investigate certain results relating to these functions, and to the theory of elliptie functions which have been given by Jacobi in two papers, "Suite des notices sur les fonctions elliptiques," Crelle, t. III. [1828] p. 306, and t. IV. [1829] p. 185, but without demonstration.

In the first place, the equation

$$
\frac{d^{2} \Sigma}{d u^{2}}-2 u\left(k^{\prime 2}-\frac{E}{K}\right) \frac{d \Sigma}{d u}+2 k k^{\prime 2} \frac{d \Sigma}{d k}=0
$$

is satisfied by $\Sigma=\Theta(u)$ or $\Sigma=H(u)$. It will be sufficient to prove this for $\Sigma=\Theta(u)$, since a similar demonstration may easily be found for the other value. The following preliminary formulæ will be required:

$$
\begin{gathered}
k \frac{d K}{d k}=\frac{E}{k^{\prime 2}}-K, \quad k \frac{d E}{d k}=E-K \\
k \frac{d K^{\prime}}{d k}=-\frac{E^{\prime}}{k^{\prime 2}}+\frac{k^{2} K}{k^{\prime 2}}, \quad K K^{\prime}-E K^{\prime}-E^{\prime} K=-\frac{1}{2} \pi
\end{gathered}
$$

which are all of them known.

Now, writing $\Theta(u)$ under the slightly more convenient form

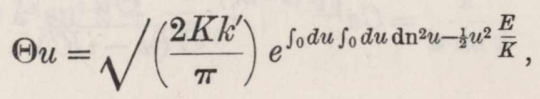

we have

$$
\begin{aligned}
& \frac{d \Theta u}{d u}=\left(\int_{0} d u \operatorname{dn}^{2} u-\frac{E}{K} u\right) \Theta u=\left\{u\left(k^{\prime 2}-\frac{E}{K}\right)+k^{2} \int_{0} d u \operatorname{cn}^{2} u\right\} \Theta u \\
& \frac{d^{2} \Theta u}{d u^{2}}=\left[\operatorname{dn}^{2} u-\frac{E}{K}+\left\{u\left(k^{\prime 2}-\frac{E}{K}\right)+k^{2} \int_{0} d u \operatorname{cn}^{2} u\right\}^{2}\right] \Theta u \\
& \frac{d \Theta u}{d k}=\left[\frac{1}{2 K k^{\prime}} \frac{d K k^{\prime}}{d k}-\frac{1}{2} u^{2} \frac{d}{d k} \frac{E}{K}+\int_{0} d u \int_{0} d u \frac{d}{d k} \mathrm{dn}^{2} u\right] \Theta u
\end{aligned}
$$


The success of the process depends upon a transformation of the double integral

$$
\int_{0} d u \int_{0} d u \frac{d}{d k} \operatorname{dn}^{2} u
$$

to effect this we have

$$
\frac{d}{d k} \operatorname{dn}^{2} u=-2 k \operatorname{sn} u\left(\operatorname{sn} u+k \frac{d}{d k} \operatorname{sn} u\right) ;
$$

but, by a known formula,

$$
k^{\prime 2} \frac{d}{d k} \operatorname{sn} u=-k \operatorname{cn} u \operatorname{dn} u \int_{0} \operatorname{cn}^{2} u d u+k \operatorname{cn}^{2} u \operatorname{sn} u ;
$$

whence

$$
\begin{aligned}
& \operatorname{sn} u+k \frac{d}{d k} \operatorname{sn} u=\frac{1}{k^{\prime 2}} \operatorname{sn} u \operatorname{dn}^{2} u-k^{2} \operatorname{cn} u \operatorname{dn} u \int_{0} d u \operatorname{cn}^{2} u \\
& \frac{d}{d k} \operatorname{dn}^{2} u=-\frac{2 k}{k^{\prime 2}}\left(\operatorname{sn}^{2} u \operatorname{dn}^{2} u-k^{2} \operatorname{sn} u \operatorname{cn} u \operatorname{dn} u \int_{0} d u \operatorname{cn}^{2} u\right) \\
&=-\frac{2 k}{k^{\prime 2}}\left\{\operatorname{sn}^{2} u \operatorname{dn}^{2} u+\frac{1}{2} k^{2}\left(\frac{d}{d u} \operatorname{cn}^{2} u\right) \int_{0} d u \operatorname{cn}^{2} u\right\}
\end{aligned}
$$

whence

$$
\begin{aligned}
\int_{0} d u \int_{0} d u \frac{d}{d k} \operatorname{dn}^{2} u & =-\frac{2 k}{k^{\prime 2}}\left\{\int_{0} d u \int_{0} d u \operatorname{sn}^{2} u \operatorname{dn}^{2} u+\frac{1}{2} k^{2} \int_{0} d u\left(\operatorname{cn}^{2} u \int d u \operatorname{cn}^{2} u-\int d u \operatorname{cn}^{4} u\right)\right\} \\
& =-\frac{k}{k^{\prime 2}}\left\{\int_{0} d u \int_{0} d u\left(2 \operatorname{sn}^{2} u \operatorname{dn}^{2} u-k^{2} \operatorname{cn}^{4} u\right)+\frac{1}{2} k^{2}\left(\int_{0} d u \mathrm{cn}^{2} u\right)^{2}\right\}
\end{aligned}
$$

But

$$
\frac{d^{2}}{d u^{2}} \operatorname{sn}^{2} u=2\left(\operatorname{cn}^{2} u \operatorname{dn}^{2} u-\operatorname{sn}^{2} u \operatorname{dn}^{2} u-k^{2} \operatorname{sn}^{2} u \operatorname{cn}^{2} u\right)=2\left(k^{\prime 2}-2 \operatorname{sn}^{2} u \operatorname{dn}^{2} u+k^{2} \operatorname{cn}^{4} u\right)
$$

or, integrating,

$$
\operatorname{sn}^{2} u=k^{\prime 2} u^{2}-2 \int_{0} d u \int_{0} d u\left(2 \operatorname{sn}^{2} u \operatorname{dn}^{2} u-k^{2} \operatorname{cn}^{4} u\right)
$$

whence at length

$$
\int_{0} d u \int_{0} d u \frac{d}{d k} \operatorname{dn}^{2} u=-\frac{1}{2} k u^{2}+\frac{1}{2} \frac{k}{k^{\prime 2}} \operatorname{sn}^{2} u+\frac{k^{3}}{2 k^{\prime 2}}\left(\int_{0} d u \mathrm{cn}^{2} u\right)^{2} .
$$

Also

$$
\frac{d}{d k} K k^{\prime}=\frac{E-K}{k k^{\prime}}, \frac{d}{d k} \frac{E}{K}=\frac{1}{k k^{\prime 2}}\left\{k^{\prime 2}\left(\frac{2 E}{K}-1\right)-\frac{E^{2}}{K^{2}}\right\},
$$

so that

$$
\frac{d \Theta u}{d k}=\frac{1}{2 k k^{\prime 2}}\left\{\frac{E}{K}-\mathrm{dn}^{2} u-u^{2}\left(k^{\prime 2}-\frac{E}{K}\right)^{2}-k^{4}\left(\int d u \mathrm{cn}^{2} u\right)^{2}\right\} \Theta u .
$$


Substituting these values of $\frac{d}{d u} \Theta u, \frac{d^{2}}{d u^{2}} \Theta u$ and $\frac{d}{d k} \Theta u$ in the equation (4) in the place of the corresponding differential coefficients of $\Sigma$, all the terms vanish, or the equation is satisfied by $\Sigma=\Theta(u)$, and similarly it would be satisfied by $\Sigma=\mathrm{H}(u)$.

Assume now

$$
\omega=\frac{\pi K^{\prime}}{K}, \quad v=\frac{\pi u}{2 \bar{K}}
$$

then observing the equation

$$
\frac{d}{d k} \frac{K^{\prime}}{K}=\frac{1}{K^{2} k k^{\prime 2}}\left(K K^{\prime}-K E^{\prime}-K^{\prime} E\right)=-\frac{\pi}{2 K^{2} k k^{\prime 2}},
$$

we have

$$
\begin{aligned}
& \frac{d \Sigma}{d u}=\frac{\pi}{2 K} \frac{d \Sigma}{d v}, \quad \frac{d^{2} \Sigma}{d u^{2}}=\frac{\pi^{2}}{4 K^{2}} \frac{d^{2} \Sigma}{d v^{2}}, \\
& \frac{d \Sigma}{d k}=\frac{v}{k k^{\prime 2}}\left(k^{\prime 2}-\frac{E}{K}\right) \frac{d \Sigma}{d v}-\frac{\pi^{2}}{2 K^{2} k k^{\prime 2}} \frac{d \Sigma}{d \omega}
\end{aligned}
$$

whence, substituting in the equation (4), this becomes

$$
\frac{d^{2} \Sigma}{d v^{2}}-4 \frac{d \Sigma}{d \omega}=0
$$

which is of course satisfied as before by $\Sigma=\Theta(u)$, or $\Sigma=H(u)$, an equation demonstrated in a different manner (by means of expansions) by Jacobi in the Memoirs referred to.

Consider next the equation

$$
\frac{d^{2} \Sigma}{d u^{2}}-2 n u\left(k^{\prime 2}-\frac{E}{K}\right) \frac{d \Sigma}{d u}+2 n k k^{\prime 2} \frac{d \Sigma}{d k}=0
$$

( $n$ being any positive integer number). Then, by assuming

$$
\omega=n \frac{\pi K^{\prime}}{K}, \quad v=\frac{n \pi u}{K},
$$

we should be led as before to the equation (5). Hence, considering $\Theta u$ or $\mathrm{H} u$ as functions of $u$ and $\frac{K^{\prime}}{K}$, the equation (6) is satisfied by assuming for $\Sigma$ a corresponding function of $n u$ and $\frac{n K^{\prime}}{K}$. Let $\lambda$ be the modulus corresponding to a transformation of the $n^{\text {th }}$ order; then $\Lambda, \Lambda^{\prime}$ being the complete functions corresponding to this modulus, $\frac{\Lambda^{\prime}}{\Lambda}=n \frac{K^{\prime}}{K}$, so that the equation (6) will be satisfied by assuming $\Sigma=\Theta,(n u)$ or $\Sigma=H_{\imath}(n u)$, where $\Theta_{\iota}, H_{l}$, correspond to the new modulus $\lambda$. 
Assume now in the equation (6),

$$
\Sigma=\left(\frac{\pi}{2}\right)^{\frac{1}{2}(n-1)}\left(K k^{\prime}\right)^{-\frac{1}{2}(n-1)} \Theta^{n} u . z .
$$

Hence, substituting,

$$
\frac{d^{2}}{d u^{2}}\left(\Theta^{n} u \cdot z\right)-2 n u\left(k^{\prime 2}-\frac{E}{K}\right) \frac{d}{d u}\left(\Theta^{n} u \cdot z\right)+2 n k k^{\prime 2}\left(K k^{\prime}\right)^{\frac{1}{2}(n-1)} \frac{d}{d k}\left[\left(K k^{\prime}\right)^{-\frac{1}{2}(n-1)} \Theta^{n} u \cdot z\right]=0
$$

but

$$
\left(K k^{\prime}\right)^{\frac{1}{2}(n-1)} \frac{d}{d k}\left[\left(K k^{\prime}\right)^{-\frac{1}{2}(n-1)} \Theta^{n} u . z\right]=\frac{d}{d k}\left(\Theta^{n} u . z\right)-\frac{n-1}{2 K k^{\prime}} \frac{d K k^{\prime}}{d k} \Theta^{n} u . z
$$

or effecting the differentiation, and eliminating $\frac{d \Theta u}{d k}$ by means of the equation obtained from (4) by writing $\Sigma=\Theta u$,

$$
\begin{aligned}
\left(K k^{\prime}\right)^{\frac{1}{2}(n-1)} \frac{d}{d k} & {\left[\left(K k^{\prime}\right)^{-\frac{1}{2}(n-1)} \Theta^{u} u \cdot z\right] } \\
& =\Theta^{n} u\left[\frac{d z}{d k}-\frac{n z}{2 k k^{\prime 2} \Theta u}\left\{\frac{d^{2} \Theta u}{d u^{2}}-2\left(k^{\prime 2}-\frac{E}{K}\right) \frac{d \Theta u}{d u}\right\}+\frac{n-1}{2 k k^{\prime 2}}\left(1-\frac{E}{K}\right) z\right] .
\end{aligned}
$$

Substituting in (6) and reducing,

$$
\begin{aligned}
\frac{d^{2} z}{d u^{2}}+2 n & {\left[\frac{1}{\Theta n} \frac{d \Theta u}{d u}-u\left(k^{\prime 2}-\frac{E}{K}\right)\right] \frac{d z}{d u}+2 n k k^{\prime 2} \frac{d z}{d k} } \\
& +n(n-1)\left\{\left[\frac{1}{\Theta^{2} u}\left(\frac{d \Theta u}{d u}\right)^{2}-\frac{1}{\Theta u} \frac{d^{2} \Theta u}{d u^{2}}\right]+\left(1-\frac{E}{K}\right)\right\} z=0, \\
\frac{d^{2} z}{d u^{2}}+2 n & {\left[\frac{d \log \Theta u}{d u}-u\left(k^{\prime 2}-\frac{E}{K}\right)\right] \frac{d z}{d u}+2 n k k^{\prime 2} \frac{d z}{d k} } \\
& +n(n-1)\left[-\frac{d^{2} \log \Theta u}{d u^{2}}+\left(1-\frac{E}{K}\right)\right] z=0 .
\end{aligned}
$$

But

$$
\begin{aligned}
& \frac{d \log \Theta u}{d u}=u\left(k^{\prime 2}-\frac{E}{K}\right)=k^{2} \int_{0} d u \mathrm{cn}^{2} u \\
& \frac{d^{2} \log \Theta u}{d u^{2}}=1-\frac{E}{K}-k^{2} \operatorname{sn}^{2} u
\end{aligned}
$$

whence

$$
\frac{d^{2} z}{d u^{2}}+2 n k^{2}\left(\int_{0} d u \mathrm{cn}^{2} u\right) \frac{d z}{d u}+2 n k k^{\prime 2} \frac{d z}{d k}+n(n-1) k^{2} \operatorname{sn}^{2} u \cdot z=0
$$

which is therefore satisfied by

$$
z=\left(\frac{2 K k^{\prime}}{\pi}\right)^{\frac{1}{2}(n-1)} \frac{\Theta, n u}{\Theta^{n} u}, \quad z=\left(\frac{2 K k^{\prime}}{\pi}\right)^{\frac{1}{2}(n-1)} \frac{\mathrm{H}_{,} n u}{\Theta^{n} u}:
$$


and each of these values is an algebraical function of sn $u$, (viz. either a rational function or a rational function multiplied by $\operatorname{cn} u \operatorname{dn} u$ ). Also, in the transformation of the $n^{\text {th }}$ order,

$$
\sqrt{ } \lambda \text { sn, } u=\frac{\mathrm{H}_{,}(n u)}{\Theta,(n u)}
$$

so that it is clear that the above values of $z$ may be taken for the denominator and numerator respectively of $\sqrt{ } \lambda \mathrm{sn}, u$; i.e. these quantities each of them satisfy the equation (7).

By assuming

$$
x=\sqrt{ } k \operatorname{sn} u, \quad \alpha=k+\frac{1}{k},
$$

this becomes

$$
n(n-1) x^{2} z+(n-1)\left(\alpha x-2 x^{3}\right) \frac{d z}{d x}+\left(1-\alpha x^{2}+x^{4}\right) \frac{d^{2} z}{d x^{2}}-2 n\left(\alpha^{2}-4\right) \frac{d z}{d \alpha}=0
$$

which is therefore satisfied by assuming for $z$ either the numerator or the denominator of $\sqrt{ } \lambda \mathrm{sn}, u$ (the transformation of the $n^{\text {th }}$ order), which is the form in which the property is given by Jacobi.

In the case where $n$ is odd, the denominator is of the form

$$
B_{0}+B, x^{2} \ldots+B_{\frac{1}{2}(n-1)} x^{n-1},
$$

and then the numerator is

$$
x\left(B_{\frac{1}{2}(n-1)} \cdots+B, x^{n-3}+B_{0} x^{n-1}\right),
$$

where



and all the remaining coefficients may be determined from these, the modular equation being supposed known. But the principal use of the formula is for the multiplication of elliptic functions, which it is well known corresponds to the case where $n$ is a square number. Writing $n=\nu^{2}$, when $\nu$ is odd, the denominator is

$$
1+B_{2} x^{4} \ldots+B_{\frac{1}{2}\left(\nu^{2}-3\right)} x^{\nu^{2}-3} \pm \nu x^{\nu^{2-1}},
$$

(the \pm sign according as $\nu=(4 p+1)$ or $(4 p-1)$ ); and the numerator is obtained from this by multiplying by $x$ and reversing the order of the coefficients. When $\nu$ is even the denominator is

$$
1+B_{2} x^{4} \cdots \pm B_{2} x^{v^{2}-4} \pm x^{\nu^{2}},
$$

( + or - , according as $\nu=4 p$ or $\nu=4 p+2$ ), so that there are only half as many coefficients to be determined; but then the numerator must be separately investigated. 
In general, by leaving $n$ indeterminate, and integrating in the form of a series arranged according to ascending powers of $x^{2}$; then, whenever $n$ is a square number, the series terminates and gives the denominator of the corresponding formula of multiplication; but the general form of the coefficients has not hitherto been discovered.

By writing $\frac{x}{\sqrt{ } n}$ instead of $x$, and then making $n$ infinite, the equation (8) takes the form

$$
x^{2} z+\alpha x \frac{d z}{d x}+\frac{d^{2} z}{d x^{2}}-2\left(\alpha^{2}-4\right) \frac{d z}{d \alpha}=0
$$

and it is worth while, before attempting the solution of the general case, to discuss this more simple one ${ }^{1}$.

Assume

$$
z=1+C_{1} \frac{x^{2}}{1.2} \ldots+C_{r} \frac{x^{2 r}}{1.2 \ldots 2 r}+\ldots
$$

then it is easy to obtain

$$
C_{r+2}=-(2 r+1)(2 r+2) C_{r}-(2 r+2) \alpha C_{r+1}+2\left(\alpha^{2}-4\right) \frac{d C_{r+1}}{d \alpha} .
$$

The general form may be seen to be

$$
C_{r}=(-)^{r+1}\left\{2^{2 r-3} C_{r}^{1} \alpha^{r-2}+2^{2 r-6} C_{r}^{2} \alpha^{r-4}+\ldots\right\},
$$

and then

$$
C_{r+1}^{p}-p C_{r}^{p}=-r(2 r-1) C_{r-1}^{p-1}+16(r+2-2 p) C_{r}^{p-1} .
$$

The complete value of $C_{r}{ }^{p}$ (assuming $C_{r}^{0}=0$ ) is given by an equation of the form

$$
C_{r}^{p}={ }^{\circ} C_{r}{ }^{p}+{ }^{1} C_{r}^{p} 2^{r}+{ }^{2} C_{r}^{p} 3^{r} \ldots+{ }^{p-1} C_{r}^{p} p^{r},
$$

where ${ }^{0} C_{r}{ }^{p},{ }^{1} C_{r} p, \ldots \ldots$ are algebraical functions of $r$ of the degrees $2 p-2,2 p-4$, \&c. respectively; but as I am not able completely to effect the integration, and my only object being to give an idea of the law of the successive terms, it will be sufficient to consider the first or algebraical term ${ }^{\circ} C_{r}{ }^{p}$, which is determined by the same equation as $C_{r}^{p}$, and is moreover completely determined by this equation and the single additional

${ }^{1}$ Writing $(\beta+2)$ for $a$, and putting $z=e^{\frac{1}{2} x^{2}} \rho$, this becomes

and if $\rho=\Sigma Z_{n} \beta^{n}$,

$$
\frac{d^{2} \rho}{d x^{2}}-\rho=\beta x^{2} \rho-\beta x \frac{d \rho}{d x}+\left(8 \beta+2 \beta^{2}\right) \frac{d \rho}{d \beta} ;
$$

$$
\frac{d^{2} Z_{n}}{d x^{2}}-(8 n+1) Z_{n}=\left(x^{2}+2 n-2-x \frac{d}{d x}\right) Z_{n-1} ;
$$

from which the successive values of $Z_{0}, Z_{1}$, \&c. might be calculated.

C. 
relation $C_{r}{ }^{1}=1$, since the arbitrary constants of the integration affect only the terms multiplied by $2^{r}, 3^{r}$, \&c.

Assume $C_{r}^{p}$

$$
=\frac{1}{[p-1]^{p-1}}\left\{2^{p-1} L^{p}[r-2]^{2 p-2}+2^{p-2} M^{p}[r-3]^{2 p-3}+\ldots 2^{-p+1} X^{p}[r-2 p]^{0}\right\} ;
$$

and substituting this value,

$$
\begin{aligned}
&(1-p) L^{p}=(1-p)\left\{L^{p-1}\right. \\
&(1-p) M^{p}-2 p(2-2 p) L^{p}=(1-p)\left\{M^{p-1}-11 L^{p-1}\right. \\
&(1-p) N^{p}-2 p(3-2 p) M^{p}=(1-p)\left\{N^{p-1}-7 M^{p-1}+12 L^{p-1}\right\} \\
&(1-p) O^{p}-2 p(4-2 p) N^{p}=(1-p)\left\{O^{p-1}-3 N^{p-1}+30 M^{p-1}\right\} \\
& \vdots
\end{aligned}
$$

the law of which is obvious, the coefficients on the second side in the $q$ th line being $1,4 q-19$, and $(2 q-3)(2 q-2)$ respectively. By successive integrations and substitutions

$$
\begin{array}{ll}
L^{p}-L^{p-1}=0, & L^{p}=1, \\
M^{p}-M^{p-1}=4 p-11, & M^{p}=(p-1)(2 p-7), \\
N^{p}-N^{p-1}=-8 p^{3}+26 p^{2}+49 p-114 ; & \vdots
\end{array}
$$

(the constants determined by $M^{1}=0, N^{1}=0, O^{2}=0, P^{2}=0, \ldots$ so as to make $C_{r}^{p}$ contain positive powers only of $r$ ).

The following are a few of the complete values of $C_{r}{ }^{p}$, the constants determined so as to satisfy $C_{p+1}{ }^{p}=0$ (except $C_{2}{ }^{1}=1$ ), and the factorials being partially developed in powers of $r$, viz.

$$
\begin{aligned}
& C_{r}{ }^{1}=1 \\
& C_{r^{2}}=(r-3)(2 r-7), \\
& C_{r}{ }^{3}=\frac{1}{2}(r-4)(r-5)\left(4 r^{2}-24 r+51\right), \\
& C_{r^{4}}=\frac{1}{6}\left\{(r-5)(r-6)(r-7)\left(8 r^{3}-60 r^{2}+286 r+63\right)+384\left(9 r^{2}-93 r+242-2.4^{r-5}\right)\right\} \\
& \& c .
\end{aligned}
$$

(it is curious that $C_{5}^{4}, C_{6}^{4}, C_{7}^{4}$, all three of them vanish). It seems hopeless to continue this investigation any further.

Returning to the equation (8), and assuming for $z$ an expression of the same form as before, we have, corresponding to the equations before found for the coefficients $C_{r}$,

$C_{r+2}=-(2 r+1)(2 r+2)(n-2 r)(n-2 r-1) C_{r}-(2 r+2)(n-2 r-2) \alpha C_{r+1}+2 n\left(\alpha^{2}-4\right) \frac{d C_{r+1}}{d \alpha}$ 
The case corresponding to the denominator in the multiplication of elliptic functions is that of $C_{0}=1, C_{1}=0$. It is easy to form the table-

$$
\begin{aligned}
C_{0}= & 1, \\
C_{1}= & 0, \\
C_{2}= & -2 n(n-1), \\
C_{3}= & 8 n(n-1)(n-4) \alpha, \\
C_{4}= & -4 n(n-1)(n-4)[n+75] \\
& -32 n(n-1)(n-4)(n-9) \alpha^{2}, \\
C_{5}= & 96 n(n-1)(n-4)(n-9) \quad[n+44] \alpha \\
& +128 n(n-1)(n-4)(n-9)(n-16) \alpha^{3}, \\
C_{6}= & -24 n(n-1)(n-4)(n-9)\left[17 n^{2}+403 n+9000\right] \\
& -960 n(n-1)(n-4)(n-9)(n-16) \quad[n+41] \alpha^{2} \\
& -512 n(n-1)(n-4)(n-9)(n-16)(n-25) \quad \alpha^{4},
\end{aligned}
$$$$
C_{7}=+96 n(n-1)(n-4)(n-9)(n-16)\left[79 n^{2}+2825 n+36180\right] \alpha
$$$$
+7168 n(n-1)(n-4)(n-9)(n-16)(n-25) \quad[n+42] \alpha^{3}
$$$$
+2048 n(n-1)(n-4)(n-9)(n-16)(n-25)(n-36) \quad \alpha^{5} \text {, }
$$

$$
\begin{array}{rlr}
C_{8}= & -48 n(n-1)(n-4)(n-9)\left[283 n^{4}-26978 n^{3}+277827 n^{2}-5491932 n+127764000\right] \\
& -3840 n(n-1)(n-4)(n-9)(n-16)(n-25) & {\left[23 n^{2}+1069 n+23436\right] \alpha^{2}} \\
& -15360 n(n-1)(n-4)(n-9)(n-16)(n-25)(n-36) & {[3 n+133] \alpha^{4}} \\
& -8192 n(n-1)(n-4)(n-9)(n-16)(n-25)(n-36)(n-49)
\end{array}
$$
\&c.

in which of course the coefficient of the highest power of $n$, in the successive coefficients $C_{r}$, is the value of $C_{r}$ obtained from the equation (8). With regard to the law of these coefficients I have found that

$$
\begin{aligned}
C_{r}=(-)^{r+1} & 2^{2 r-3} n\left(n-1^{2}\right) \ldots\left\{n-(r-1)^{2}\right\} C_{r}^{r^{1}} \alpha^{r-2} \\
& +2^{2 r-6} n\left(n-1^{2}\right) \ldots\left\{n-(r-2)^{2}\right\} C_{r^{2}} \alpha^{r-4} \\
& +2^{2 r-9} n\left(n-1^{2}\right) \ldots\left\{n-(r-3)^{2}\right\} C_{r}^{3} \alpha^{r-6} \\
& +\& \mathrm{c} .
\end{aligned}
$$

(where however the next term does not contain, as would at first sight be supposed, the factor $\left.n\left(n-1^{2}\right) \ldots\left\{n-(r-4)^{2}\right\}\right)$. And then

$$
\begin{aligned}
& C_{r}{ }^{1}=1, \\
& \begin{aligned}
C_{r}{ }^{2}=(r-3)[n(2 r-7) & +(r-1)(8 r-7)], \\
C_{r}{ }^{3}=\frac{1}{2}(r-4)(r-5)[ & n^{2}\left(4 r^{2}-24 r+51\right) \\
& +n\left(32 r^{3}-220 r^{2}+412 r-255\right) \\
& \left.+2(r-1)(r-2)\left(32 r^{2}-88 r+51\right)\right] .
\end{aligned}
\end{aligned}
$$


In conclusion may be given the following results, in which, recapitulating the notation

$$
x=\sqrt{ } k \text { sn } u, \quad \alpha=k+\frac{1}{k}, \quad \Delta x=\sqrt{ }\left(1-\alpha x^{2}+x^{4}\right),
$$

$\sqrt{ } k$ sn $2 u=\frac{2 x \Delta x}{1-x^{4}}$

$\sqrt{ } k$ sn $3 u=\frac{x\left(3-4 a x^{2}+6 x^{4}-x^{8}\right)}{1-6 x^{4}+4 \alpha x^{6}-3 x^{8}}$,

$\sqrt{ } k$ sn $4 u=\frac{4 x \Delta x\left(1-x^{4}\right)\left(1-2 \alpha x^{2}+6 x^{4}-2 \alpha x^{6}+x^{8}\right)}{1-20 x^{4}+32 \alpha x^{6}-\left(26+16 \alpha^{2}\right) x^{8}+32 \alpha x^{10}-20 x^{12}+x^{16}}$,

$\sqrt{ } k$ sn $5 u=x\left\{5-20 \alpha x^{2}+\left(62+16 \alpha^{2}\right) x^{4}-80 \alpha x^{6}-105 x^{8}+360 \alpha x^{10}-\left(300+240 \alpha^{2}\right) x^{12}\right.$

$$
\begin{array}{r}
\frac{\left.+\left(368 \alpha+64 \alpha^{3}\right) x^{14}-\left(125+160 \alpha^{2}\right) x^{16}+140 \alpha x^{18}-50 x^{20}+x^{24}\right\}}{\left\{1-50 x^{4}+140 \alpha x^{6}-\left(125+160 \alpha^{2}\right) x^{8}+\left(368 \alpha+64 \alpha^{3}\right) x^{10}-\left(300+240 \alpha^{2}\right) x^{12}\right.} \\
\left.+360 \alpha x^{14}-105 x^{16}-80 \alpha x^{18}+\left(62+16 \alpha^{2}\right) x^{20}-20 \alpha x^{22}+5 x^{24}\right\}
\end{array}
$$

\&c.

Thus, writing $-x^{2}$ for $x^{2}, k=1$, and therefore $\alpha=2$,

$$
\tan 3 u=x \frac{\left(3+8 x^{2}+6 x^{4}-x^{8}\right)}{1-6 x^{4}-8 x^{6}-3 x^{8}}=\frac{x\left(3-x^{2}\right)\left(1+x^{2}\right)^{3}}{\left(1-3 x^{2}\right)\left(1+x^{2}\right)^{3}}=\frac{x\left(3-x^{2}\right)}{1-3 x^{2}},
$$

where $x=\tan u$. (And in general in reducing $\tan n u$ the extraneous factor in the numerator and denominator is $\left(1+x^{2}\right)^{\frac{1}{2} n(n-1)}$.) 